\title{
Research on path planning of mobile robot based on improved artificial potential field
}

\author{
Wang Rui ${ }^{1, a}$, Wang Jinguo ${ }^{2, b^{*}}$, Wang $\mathrm{Na}^{3, \mathrm{c}}$ \\ ${ }^{1}$ Department of Information Engineering, Jilin Business and Technology College, \\ China \\ ${ }^{2}$ Department of Urology, the First Hospital of Jilin University, China \\ ${ }^{3}$ Department of Anaesthesiology, the First Hospital of Jilin University, China

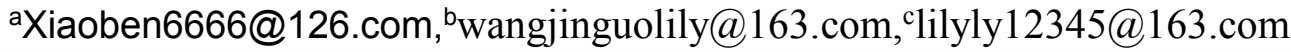

\section{Key words: Mobile robot, Path planning, artificial potential field}

\begin{abstract}
This paper mainly studies the robot path planning algorithm based on the improved artificial potential field. In the traditional artificial potential field, calculate the intensity and minimum of the potential field with the superimposed sum of Potential field strength each other and mark this point. A global optimization path is formed by the point which corresponding a series of minimum values. Finally, the practice proves that the improved algorithm can form a optimal path.
\end{abstract}

\section{Introduction}

Robot path planning problem is an important problem in robot research field. It is also the basis and core of the robot navigation[1]. Robot path planning is to seek an optimal path from starting point to the target point for mobile robot, while ensuring mobile robot and obstacles or other robots cannot collide. There are two main types of robot path planning: global path planning and local path planning[2]. At present, the existing global path planning algorithm is heuristic graph search algorithm, artificial potential field method, the visibility graph method, genetic algorithm and so on. These methods have their advantages, but there are also some limitations. It is an effective way to improve the path planning performance according to different environment features. It requires introduces new algorithm[3].

Among the numerous robot path planning algorithms, artificial potential field algorithm is a mature and more productive planning algorithm in the traditional algorithm. Artificial potential field method is a virtual method, which is proposed by Khatib[4]. Its basic idea is that the robot's moving environment is designed an abstract artificial force field. When the robot is moving in this environment, it receives the constraint of "gravity" and "repulsive ".The target node is generated by robot, and the obstacle is repulsive to the robot. Finally, the robot is controlled by the resultant of forces. Although this algorithm can produce a smooth and safe path, but artificial potential field method can produce the local optimal solution easily and produce deadlock phenomenon[5]. Therefore, how to design the robot's gravity field is the key step to solve the algorithm.

\section{Problem description}

Traditional artificial potential field model.In the artificial potential field, we take the mobile robot as a point, and it moves in the two dimensional space. The arbitrary position of the robot in the two-dimensional space is: $X\left(X=[x, y]^{T}\right)$. The direction of the robot's movement is determined 
by the joint forces of the attraction of the destination node and the repulsion of the obstacle. In artificial potential field, the attraction function of robot is:

$$
U_{a}(X)=\frac{1}{2} k\left\|X-X_{a}\right\|^{2}
$$

The attraction is the negative gradient of the target gravitational potential function. That is :

$$
F_{a}(X)=-\operatorname{grad}\left[U_{a}(X)\right]
$$

In which, $\mathrm{k}$ is the attractive position coefficient. $\mathrm{X}$ is the robot current position vector. $\mathrm{Xa}$ is a destination position vector. $\left\|X-X_{a}\right\|$ is the euclidean distance between robot and destination node. The repulsion function of the restricted area is defined as:

$$
U_{i}(X)=\left\{\begin{array}{cc}
\frac{1}{2} \eta\left[\frac{1}{\rho_{i}}-\frac{1}{\rho_{0}}\right]^{2} & \rho_{i}<=\rho_{0} \\
0 & \rho_{i}>\rho_{0}
\end{array}\right.
$$

The corresponding repulsion function is:

$$
F_{i}(X)=-\operatorname{grad}\left[U_{i}(X)\right]= \begin{cases}\eta_{i}\left[\frac{1}{\rho_{i}}-\frac{1}{\rho_{0}}\right] \frac{1}{\rho_{i}^{2}} \frac{\partial \rho_{i}}{\partial X} & \rho_{i}<=\rho_{0} \\ 0 & \rho_{i}>\rho_{0}\end{cases}
$$

In which, $\eta_{i}$ is a repulsion position gain coefficient. $\rho_{0}$ is the maximum distance of restricted area. The $\mathrm{i}$ is restricted area number and $\mathrm{i}$ is between 1 and $\mathrm{N} . \mathrm{N}$ is total number of restricted areas. $\rho_{1}$ is the shortest euclidean distance for the robot from the limited area i. Therefore, the robot force is:

$$
F_{T}(X)=F_{a}(X)+\sum_{i=1}^{N} F_{i}(X)
$$

The disadvantages of the original algorithm. If the walking environment of the robot is $U$ obstacle, the robot is influenced with attraction and repulsion force at the same time. It will shock in a certain point and can not reach the target node. As the robot did not get the whole the information environment, it can not get rid of the local minimum point. If you enter the global information environment advanced, the robot will walk the path as planning. Once the environmental information is changed, we will re enter the information into the robot. If a certain environment changes frequently, the workload of this method is great, the time of the algorithm is longer.

\section{Improvement of algorithm}

In order to avoid local minimal value in the local environmental information, we introduce the follow_wall algorithm, which allows the robot to walk along the edge of the obstacle. 
Follow_wall behavior. The basic idea of follow_wall is: assuming the robot is moving in a certain direction, calculates the next step position $\mathrm{D}[\mathrm{i}]$. According to the data from sensor, calculates the minimum distance $\mathrm{L}[\mathrm{i}]$ between obstacle and $\mathrm{D}$. After calculating all the $\mathrm{D}[\mathrm{i}]$ and the corresponding L[i], we select the minimum distance L[i] , and mark the minL. The minL should meet $\operatorname{minL}>1,1$ is the minimum distance between the robot and obstacle. If the minL of the satisfied condition exists, the robot should move towards the $\mathrm{D}$ [i] direction of the minL. If there is no minL, it means that no matter how the robot moves, it can not keep a certain minimum distance from the obstacle, then the robot remains fixed.

This algorithm is mainly used to solve local minimal value in the robot path planning, so when we judge the current robot in local minimal value, we use this algorithm. The algorithm activation condition is as follows:

$$
\begin{aligned}
& \left.\arg \left(\frac{G-P}{D}\right)>-\frac{3}{4} \pi\right\rangle \cap\left(d_{1}>0\right) \cap\left(d_{2}>0\right) \\
& \left.\arg \left(\frac{G-P}{D}\right)>\frac{3}{4} \pi\right\rangle \cap\left(d_{9}>0\right) \cap\left(d_{10}>0\right)
\end{aligned}
$$

The follow_wall exit condition corresponds the state the robot escapes the local minimal value.

$$
\arg \left(\frac{G-P}{D}\right) \in\left(-\frac{3}{4} \pi, \frac{3}{4} \pi\right) \cap\left(d_{5}<0\right) \cap\left(d_{6}<0\right)
$$

In which, $\arg (\mathrm{X})$ is the argument of vector $\mathrm{X}$. The $\mathrm{G}$ is the position of the target points. $\mathrm{P}$ is the robot current position and expressed with the vector. The $\mathrm{D}$ is the robot's current movement direction. $d_{1}$ is the readings of sensor. If $d_{1>0}$, it is have obstacles in direction of the sensor $\mathrm{S} 1$, otherwise the sensor output $d_{1}=-1$.

\section{Simulation experiment}

In this paper, a simulation experiment is done on the artificial potential field based on the follow-wall. The simulation requires that there are a shortest and no obstacle path for robot from the starting point to the target point. Firstly, we should fit some geometric parameters for the robot. The robot is considered as a particle. The radius is 5 . The initial direction is $\theta$. The maximum speed is 2 . The maximum detection range of sonar sensors is 20. The minimum distance the robot from obstacle is 1 . The results of the simulation are shown in the following figure 1 . 


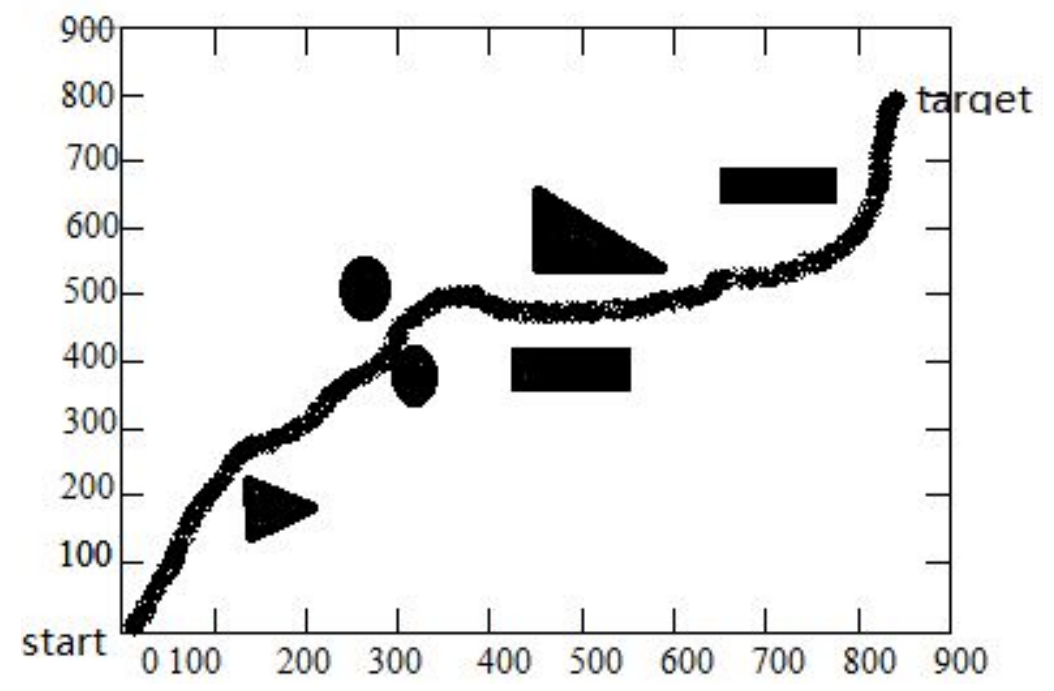

Fig 1.the path planning of the improved artificial potential field

\section{Conclusion}

Because of the local minimum of artificial potential field, we propose the follow-wall method. By judging the robot into the local minimal point, we activate this method. This will make the robot moves along the edge of the obstacle. So it can jump out of local minimum. Experiments show that this method is effective.

\section{References}

[1] Prahlad Vadakkepat,Kay Chen Tan,Wang Ming-Liang.Evolutionary artificial potential fields and their application in real time robot path planning. Proceedings of the 2000 Congress on Evolutionary Computation . 2000.

[2] L.Huang.Velocity planning for a mobile robot to track a moving target a potential field approach. Robotics and Autonomous Systems.2009.

[3] Dorigo M,Maniezzo V,Colorni A.Ant system: optimization by a colony of cooperating agents. IEEE Transactions on Systems Man and Cybernetics . 1996.

[4] Min Gyu Park,Jae Hyun Jeon,Min Cheol Lee.Obstacle avoidance for mobile robots using artificial potential field approach with simulated annealing. IEEE International Symposium on Industrial Electronics . 2001.

[5] Ge S S,Cui Y J.New potential functions for mobile robot path planning. IEEE Transactions on Robotics and Automation . 2000. 